\title{
Association of two COVID-19 outbreaks with meteorological parameters in Beijing, China
}

\author{
Yue Gao ${ }^{1}$ Ruidi Zhang ${ }^{1}$ Hongbin $\mathrm{Cao}^{1, *}$ \\ ${ }^{1}$ Faculty of Geographical Science, Beijing Normal University, Beijing 100875, China \\ ${ }^{*}$ Corresponding author.Email: caohongbin@bnu.edu.cn
}

\begin{abstract}
Since December 2019, COVID-19 has become a public health emergency of international concern, from local area to the current worldwide pandemic. Since March this year, the epidemic in Chinese cities has been effectively controlled, and the number of newly confirmed local cases in many places has reached zero growth. However, it is worth noting that some Chinese cities represented by Beijing have undergone secondary local transmission of COVID-19. According to epidemiological and laboratory studies, meteorological factors are key factors affecting the spread of infectious diseases such as coronavirus and influenza, but the impact of meteorological factors on the spread of COVID-19 virus remains controversial. This study explored the correlation between daily meteorological data in Beijing and the increase in the number of COVID-19 cases, and compared three Chinese cities with similar conditions (Jilin, Dalian and Urumqi) to explore the role of meteorological factors in the spread of COVID-19 virus. Szpilman and Kendall correlation coefficients were used to study the correlation between COVID-19 and meteorological factors. The results showed that in the first round of COVID-19 transmission, meteorological factors were significantly correlated with the development of COVID-19 $(p<0.01)$ : temperature and wind speed were positively correlated with the increase in the number of cases, while air pressure and relative humidity were negatively correlated. In the second round of epidemic spread, the impact of meteorological factors was not significant. This study is of great significance for enhancing the understanding of the current situation of COVID-19 transmission and developing relevant prevention and control measures to prevent a second outbreak of COVID-19.
\end{abstract}

Keywords(Bold): COVID-19, Meteorological parameters, Correlation analysis , Beijing.

\section{气象因子对北京市两次新冠肺炎疫情的影响}

\section{高越 ${ }^{1}$ 张瑞迪 $^{1}$ 曹红斌 ${ }^{1, *}$}

\author{
${ }^{1}$ 北京师范大学地理科学学部, 北京 100875 \\ *通讯作者. 电子邮箱: caohongbin@bnu.edu.cn
}

\begin{abstract}
摘要
自 2019 年 12 月以来，新型冠状病毒肺炎（简称“新冠肺炎”）从局部地区最初发现到现在世界范围的大流行， 已成为国际关注的突发公共卫生事件。我国各城市的疫情在今年 3 月份之后得到了有效控制, 多地最新确诊 的本土病例实现零增长。但值得注意的是, 我国以北京为代表的部分城市出现了新冠肺炎疫情本土二次传播 的情况。根据流行病学和实验室研究结果可知, 气象因子是影响冠状病毒和流感等传染病传播的关键因素, 但气象因子对新冠肺炎病毒传播的影响仍存在争议。本研究通过探究北京市逐日气象数据与新冠肺炎病例数 增长之间的关联，并对比国内类似情况的 3 个城市（吉林、大连、乌鲁木齐），探讨气象因子在新冠肺炎病 毒传播中的作用。采用斯皮尔曼 (Spearman) 和肯德尔 (Kendal1) 相关系数研究新冠肺炎疫情与气象因子的 相关性。结果表明：在第一轮新冠肺炎疫情传播中，气象因子与新冠疫情的发展显著相关（p<0.01）：其中 温度和风速与病例数增长呈正相关，气压和相对湿度则与之负相关; 而在第二轮的疫情传播中气象因子的影
\end{abstract}


响则不具有显著性。本研究对于加强对新冠肺炎传播现状的认识，并制定相关防控措施以防止疫情二次懪发 具有重要意义。

关键字：新冠肺炎，气象因子，相关分析，北京.

\section{1. 引言}

2019 年 12 月, 新型冠状病毒肺炎（简称”新冠肺 炎”, COVID-19）在湖北武汉最初被发现, 并迅速在 全球范围内流行，造成了大量人员伤亡和社会损失， 成为 “国际关注的突发公共卫生事件” (Public Health Emergency of International Concern, PHEIC) ${ }^{[1]}$ 。2020 年 3 月 11 日, 世界卫生组织 (WHO ) 宣布新冠肺炎疫情已经 “全球大流行 (pandemic) ” ${ }^{[2]}$, 这是首个冠状病毒传播引发的全球大流行。截 至 2020 年 8 月 25 日, 全球已有超过 2369 万人感染 新冠肺炎, 死亡人数超过 81 万人 ${ }^{[3]}$ 。

新冠肺炎是由新型冠状病毒 (SARS-CoV-2, 2019-nCov）感染引起的, 这是一种可能源自蝙蝠的 单链 RNA 病毒, 新型冠状病毒属 $\beta$ 属冠状病毒, 与 SARS-Cov 和 MERS-Cov 有一定的同源性; 其传染性极 强, 确诊感染者和无症状感染者都可传播, 在潜伏 期即有传染性。经呼吸道飞沫和密切接触传播是新 冠病毒主要的传播途径, 接触病毒污染的物品也可 造成感染。在相对封闭的环境中长时间暴露于高浓 度气溶胶情况下, 也存在经气溶胶传播的可能。并 且由于在粪便、尿液中可分离到新型冠状病毒, 应 注意其对环境污染造成接触传播或气溶胶传播 ${ }^{[4]}$ 。

疫情爆发后, 我国除武汉外的其他地区的疫情 在短暂的本土传播后均得到了有效控制, 新增确诊 病例数逐渐下降, 于 2020 年 3 月份首次实现本土确 诊病例零增长。但值得注意的是, 我国部分地区疫 情有所反弹, 以北京为代表的部分城市 (包括吉林、 大连、乌鲁木齐等) 在一段时间的新冠肺炎本土确 诊病例新增为零的情况下, 都出现了新一轮的新冠 肺炎疫情本土传播。

在既往的流行病学研究中, 气象因素已被证实 是影响传染病传播的重要变量之一, 特别是流感、 严重急性呼吸综合征 (severe acute respiratory syndrome, SARS) 等呼吸道传染病 ${ }^{[5-7]}$ 。流感的传播 通常都会在天气寒冷干燥的冬春季节增强 ${ }^{[6]}$ 。实验 室和流行病学研究均表明, 环境温度是影响冠状病 毒如 SARS-Cov 和 MERS-Cov 传播和存活的关键因素 ${ }^{[5}$;
${ }^{8]}$; 太阳辐射可以通过灭活 MERS-Cov 和 SARS-Cov 类 冠状病毒来阻止传播 ${ }^{[9]}$ 。

然而, COVID-19 的传播是否像其他季节性病毒 一样受到气象因子的影响仍存在争议。最近的几项 研究调查了气象因子对全球不同地区 COVID-19 传播 的影响, 如中国 ${ }^{[10-13]}$, 新加坡 ${ }^{[14]}$ 伊朗 ${ }^{[15]}$, 西班牙 ${ }^{[16]}$, 美国 ${ }^{[17]}$ ，墨西哥 ${ }^{[18]}$ ，土耳其 ${ }^{[19]}$ ，印度尼西亚 ${ }^{[20]}$ 、挪 威 ${ }^{[21]}$ 以及全球范围 ${ }^{[22 ;}{ }^{23]}$, 得到的结论各不相同, 尚 需更长时间周期和更广地区范围的数据支持。

本研究通过北京市两轮疫情传播中逐日气象数 据与新冠肺炎病例数增长之间的相关性分析, 以斯 皮尔曼秩相关系数和肯德尔等级相关系数为评价指 标, 探究气象因子对北京市两轮疫情传播中的影响。 并对比国内类似情况的 3 个城市（吉林、大连、乌 鲁木齐），探讨气象因子在新冠疫情传播中的作用。 研究对于加强对新冠肺炎传播现状的认识, 并制定 相关防控措施以防止疫情二次懪发具有重要意义。

\section{2. 数据与方法}

\section{1. 研究地简介}

北京市 $\left(115.7^{\circ}-117.4^{\circ} \mathrm{E}, 39.4^{\circ}-41.6^{\circ} \mathrm{N}\right)$ 地处华北大平原的北部, 东面与天津市毗连, 其余 均被河北省环绕。北京辖区总面积为 $16410 \mathrm{~km}^{2}$, 地 势西北高、东南低。西部、北部和东北部三面环山, 山区面积约占总面积的 $62 \%$, 东南部是一片缓缓向渤 海倾斜的平原 ${ }^{[24]}$ 。

北京的气候为暖温带半湿润半干旱季风气候, 夏季高温多雨, 冬季寒冷干燥, 春、秋短促。全年 降水的季节和空间分配都很不均匀，全年降水的 $80 \%$ 集中在夏季 $6 、 7 、 8$ 三个月, 东北部和西南部山前 迎风坡地区为相对降水中心。

\section{2. 数据来源}

新冠肺炎疫情的二级数据来自北京市卫生健康 员会官方网站 (http://wjw. bei jing. gov.cn/)。获 
取 2020 年 1 月 20 日至 8 月 25 日的每日新增感染病 例和累计感染病例, 纳入本研究。

日常基本气象因子的数据, 包括最高温度、平均 温度、最低温度, 平均相对湿度、最小相对湿度, 最 高气压、平均气压、最低气压, 极大风速、最大风速、 平均 2 分钟风速, 平均水汽压和日照时数。数据均 来自中国气象数据网 (http://data. cma. cn/) 的中 国地面气候资料日值数据集。

\section{3. 统计方法}

本研究使用斯皮尔曼等级相关系数 (Spearman's rank correlation coefficient) 和 肯德尔秩相关系数 (Kendall tau rank correlation coefficient）来检验新冠肺炎疫情与气象因子之间 的相关性。

在统计学中, 斯皮尔曼等级相关系数经常用希 腊字母 $\rho$ （rho）表示, 其值可用于衡量两个变量之 间的关联强度。Spearman 等级相关系数在实际应用 中可以用公式(1)表示:

$$
\rho=1-6 \times \frac{\sum d_{i}^{2}}{n\left(n^{2}-1\right)}
$$

式中 $d_{i}$ 表示两个变量的等级差数, $n$ 表示等级个 数。 $\rho$ 的值为 +1 和 -1 时分别为完全正相关和负相关。

肯德尔秩相关系数可以用希腊字母 $\tau$ （tau）表示 其值, 它常用于衡量两个变量之间的有序关联。在 实际应用中，其估计公式如下：

$$
\tau=\frac{[(\text { concor })-(\text { discor })]}{\frac{1}{2} \times n \times(n-1)}
$$

式中, concor 和 discor 分别表示协调对和不协调 对的数量。 $\mathrm{n}$ 表示对的数量。值的范围从 -1 到 +1 分 别为完全正相关和负相关。

在本研究中, 我们使用 IBM SPSS23.0 统计分析 软件进行上述统计计算。考虑到新冠病毒的传播效 率, 我们定义一轮完整的本土疫情传播时间段为新 增病例不为零时间段加上该时段前后七日的时间。

\section{3. 结果与讨论}

\section{1. 北京市两轮疫情传播与气象因子的相关 性}

北京市第一轮疫情传播时间段为 2020 年 1 月 13 日至 3 月 30 日，第二轮疫情传播时间段为 2020 年 6 月 4 日至 8 月 13 日。

\subsection{1. 各参数变化特征}

2020 年 1 月 20 日，北京市正式报告了本地首例 新冠肺炎病例。截至 2020 年 8 月 25 日, 北京市累计 报告新冠肺炎病例 759 例, 其中第一轮疫情 416 例, 第二轮 343 例。图 1 显示北京市 2020 年 1 月 13 日至 8 月 13 日新冠肺炎日新增病例和累计病例数。由于 在新冠肺炎传播初期, 北京市就迅速采取了严格而 有效的疫情防控措施, 故北京市在两轮疫情传播中 单日新增病例数最多分别为 29 例和 34 例。

图 1 显示了北京市不同气象因子的日变化。第 一轮和第二轮疫情的每日温度区间分别为 $-9.4^{\circ} \mathrm{C}$ $\sim 26.2^{\circ} \mathrm{C}$ 和 $17.1^{\circ} \mathrm{C} \sim 37.8^{\circ} \mathrm{C}$, 气温上升明显。与在波动 中上升的气温不同, 两轮疫情期间的日均气压变化 幅度不大, 期间平均气压分别为 $1019.72 \mathrm{hPa}$ 和 $1000.63 \mathrm{hPa}$ 。相对湿度除了由空气中水蒸气含量所 决定, 还受到环境的温度和气压的影响, 它是实际 水汽压与当前温度下饱和水汽压的比值。研究期间, 北京市的平均相对湿度波动范围分别为 $13.8 \%$ $92.8 \%$ 和 $24.0 \%$ $87.3 \%$ 。研究期间的平均风速也保持在一 个较为稳定的数值范围内, 分别是 $2.06 \mathrm{~m} / \mathrm{s}$ 和 $1.93 \mathrm{~m} / \mathrm{s}$ 。

\subsection{2. 相关性分析}

北京市第一轮疫情的每日新增病例数和气象因 子的日值数据之间的相关性分析结果如表 1 所示。 在第一轮疫情传播中, 温度和风速与新增病例数呈 显著负相关, 相对湿度和气压与新增病例数呈显著 正相关。但在第二轮疫情传播中, 大部分气象因子 与日新增病例数之间没有显著相关性（表 2)

这可能是由于第一轮疫情传播初期，居民对病 毒认知不够、防疫物资准备不足, 医院的诊疗手段 和隔离措施不充分等等, 在这种没有或缺少防控措 施的原始传播条件下, 较低的温度和风速, 较高的 湿度和气压更有利于病毒的传播。而在第二轮疫情 爆发时, 疫情防控已经常态化, 居民自我保护意识 较强, 并且检测排查迅速; 可以认为, 在第二轮疫 情传播中, 其他人为因素对病毒传播的影响大于气 象因素的影响。 

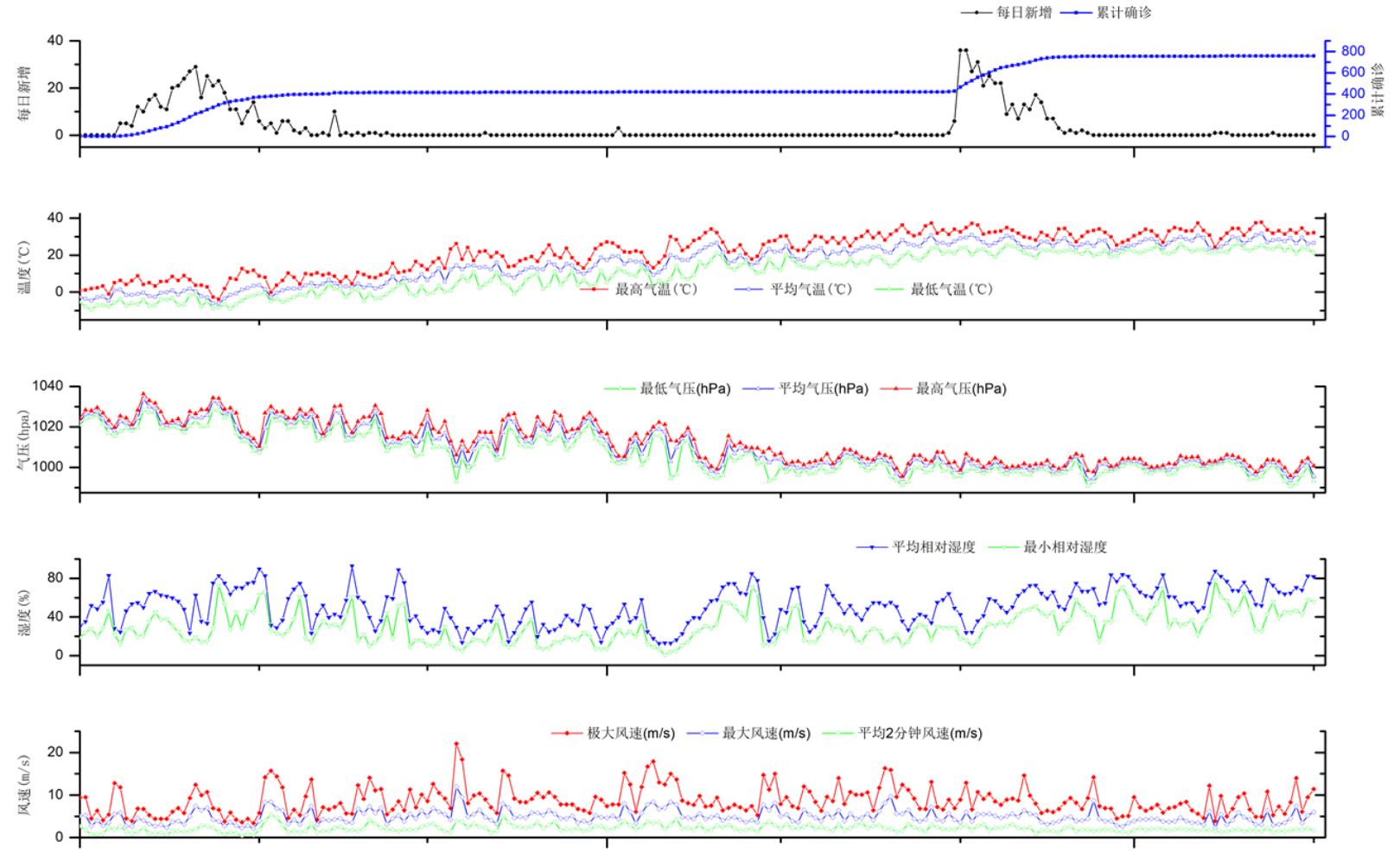

一一旦照时数( $(\mathrm{h})$ 一一平均水气压 $(\mathrm{hPa})$

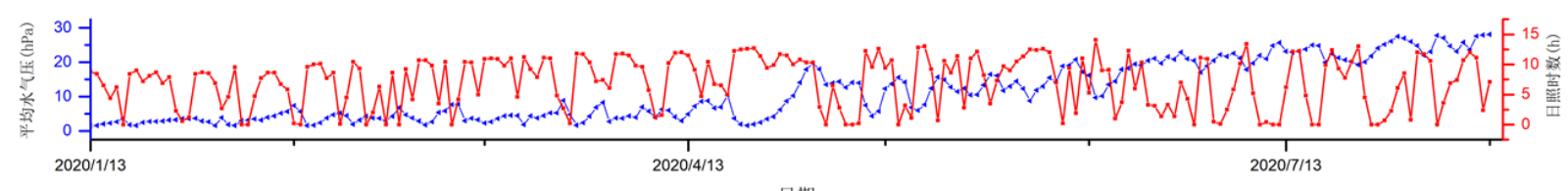

表 1 北京市新冠疫情与气象因子的相关性分析结 果 (0113-0330, $\mathrm{n}=78$ )

\begin{tabular}{|c|c|c|c|c|}
\hline \multirow{2}{*}{} & \multicolumn{2}{|c|}{ Kendall } & \multicolumn{2}{c|}{ Spearman } \\
\cline { 2 - 5 } & $\tau$ & $\mathrm{p}$ & $\rho$ & $\mathrm{p}$ \\
\hline 最高气温 $\left({ }^{\circ} \mathrm{C}\right)$ & $-0.384^{* *}$ & 0.000 & $-0.488^{* *}$ & 0.000 \\
\hline 平均气温 $\left({ }^{\circ} \mathrm{C}\right)$ & $-0.479^{* *}$ & 0.000 & $-0.588^{* *}$ & 0.000 \\
\hline 最低气温 $\left({ }^{\circ} \mathrm{C}\right)$ & $-0.425^{* *}$ & 0.000 & $-0.538^{* *}$ & 0.000 \\
\hline 最低气压 $(\mathrm{hPa})$ & $0.384^{* *}$ & 0.000 & $0.500^{* * *}$ & 0.000 \\
\hline 平均气压 $(\mathrm{hPa})$ & $0.353^{* *}$ & 0.000 & $0.470^{* *}$ & 0.000 \\
\hline 最高气压 $(\mathrm{hPa})$ & $0.316^{* *}$ & 0.000 & $0.425^{* *}$ & 0.000 \\
\hline 平均相对湿度 $(\%)$ & $0.311^{* *}$ & 0.000 & $0.409^{* * *}$ & 0.000 \\
\hline 最小相对湿度 $(\%)$ & $0.211^{*}$ & 0.012 & $0.291^{* *}$ & 0.010 \\
\hline 极大风速 $(\mathrm{m} / \mathrm{s})$ & $-0.205^{*}$ & 0.015 & $-0.294^{* *}$ & 0.009 \\
\hline 最大风速 $(\mathrm{m} / \mathrm{s})$ & $-0.210^{*}$ & 0.013 & $-0.298^{* *}$ & 0.008 \\
\hline 平均 2 分钟风速 $(\mathrm{m} / \mathrm{s})$ & $-0.235^{* *}$ & 0.006 & $-0.319^{* *}$ & 0.004 \\
\hline 平均水气压 $(\mathrm{hPa})$ & -0.098 & 0.247 & -0.121 & 0.293 \\
\hline 日照时数 $(\mathrm{h})$ & $-0.177^{*}$ & 0.035 & $-0.229^{*}$ & 0.044 \\
\hline
\end{tabular}

** Correlation is significant at the 0.01 level (2-tailed).

* Correlation is significant at the 0.05 leve1 (2-tailed).
表 2 北京市新冠疫情与气象因子的相关性分析结 果 (0604-0813, n=71)

\begin{tabular}{|c|c|c|c|c|}
\hline \multirow{2}{*}{} & \multicolumn{2}{|c|}{ Kendall } & \multicolumn{2}{c|}{ Spearman } \\
\cline { 2 - 5 } & $\tau$ & $\mathrm{p}$ & $\rho$ & $\mathrm{p}$ \\
\hline 最高气温 $\left({ }^{\circ} \mathrm{C}\right)$ & -0.057 & 0.527 & -0.077 & 0.525 \\
\hline 平均气温 $\left({ }^{\circ} \mathrm{C}\right)$ & -0.035 & 0.700 & -0.040 & 0.74 \\
\hline 最低气温 $\left({ }^{\circ} \mathrm{C}\right)$ & -0.061 & 0.499 & -0.081 & 0.500 \\
\hline 最低气压 $(\mathrm{hPa})$ & -0.041 & 0.651 & -0.067 & 0.578 \\
\hline 平均气压 $(\mathrm{hPa})$ & -0.089 & 0.325 & -0.133 & 0.269 \\
\hline 最高气压 $(\mathrm{hPa})$ & -0.066 & 0.464 & -0.093 & 0.440 \\
\hline 平均相对湿度 $(\%)$ & $-0.193^{*}$ & 0.033 & $-.245^{*}$ & 0.039 \\
\hline 最小相对湿度 $(\%)$ & -0.108 & 0.233 & -0.139 & 0.248 \\
\hline 极大风速 $(\mathrm{m} / \mathrm{s})$ & 0.142 & 0.117 & 0.184 & 0.124 \\
\hline 最大风速 $(\mathrm{m} / \mathrm{s})$ & 0.140 & 0.124 & 0.175 & 0.144 \\
\hline 平均2 分钟风速 $(\mathrm{m} / \mathrm{s})$ & 0.141 & 0.127 & 0.194 & 0.106 \\
\hline 平均水气压 $(\mathrm{hPa})$ & $-0.325^{* *}$ & 0.000 & $-0.402^{* *}$ & 0.001 \\
\hline 日照时数 $(\mathrm{h})$ & -0.100 & 0.273 & -0.146 & 0.224 \\
\hline
\end{tabular}

图 1 北京市 COVID-19 和气象因子日变化（2020.0113-2020. 0813） 


\section{2. 同类三市两轮疫情相关性分析对比}

为验证结论是否可靠, 选取国内出现过第二次 疫情本土传播的另外三个城市 (吉林、大连、乌鲁 木齐）做同样的相关性分析，与北京的结果作对比。 三个城市的两轮疫情传播过程中, 日新增病例与气 象因子之间基本不存在显著的相关性。特别是更接 近自然传播条件的第一轮疫情, 也没有表现出显著 相关性。

对比四个城市的第一轮疫情持续时间和累计病 例数 (图 2), 吉林、大连和乌鲁木齐三个城市不仅 累计病例总数远少于北京, 疫情传播的持续时间也 较短; 使用较小的样本量分析得到的结果难以支撑 强有力的结论, 故不能给出疫情传播与气象因子之 间是否有相关性的准确推论。

\section{3. 同类研究分析结果对比}

气象因子作为影响多种传染病传播的重要影响 因素, 在这次新冠疫情中自然受到了很大关注, 国 内外学者已经针对新冠疫情传播与气象因子之间的 关系开展了一系列研究。

\subsection{1 温度}

温度作为影响病原体活性的重要因素, 可以在 涉及流行病发展、预防和控制的公共卫生方面发挥 重要作用 ${ }^{[16 ;}$; 25$]$ 。环境温度与 SARS-CoV-1 传播的负相 关关系在以往的研究中已经得到证实 ${ }^{[7]}$ 。在本研究 中, 近似原始传播条件下, 温度与北京市新增病例 数呈显著负相关。一项针对中国除武汉之外的 30 个 省会城市的 meta 分析研究也得到了类似的结论, 即 较低的温度可能有利于新冠病毒的传播 ${ }^{[11]}$ 。Shi 等人 针对中国大陆 31 个省级地区的研究也表明温度与新 冠的流行呈显著负相关 ${ }^{[12]}$ 。相反, 一项针对中国 122 个城市 (包括武汉) 的研究认为日均气温与日新增病 例数呈线性正相关 $(p<0.01)^{[10]}$ 。与上述两项研究 的结论不同的是, Yao 等分析了中国 224 个城市的数 据后, 得出了新冠疫情的传播与温度变化没有关系 的结论 ${ }^{[13]}$ 。

在国外的研究中, Tosepu 等人 ${ }^{[20]}$ 和 Pani $\mathrm{i}^{[14]}$ 等 人分别在印度尼西亚雅加达和新加坡的相关性分析 均得到了新冠肺炎的大流行与平均气温是显著正相 关的结论。然而 Mendez-Arriaga (2020) 基于墨西哥 首都和 31 个州的数据, 发现温度与新冠肺炎的日新 增病例负相关, 并且认为热带气候可以延缓本地传 播的开始。一项针对西班牙巴塞罗那地区的研究也 证实了环境温度和新冠传播之间的负相关关系 ${ }^{[16]}$ 。 Holtmann 等人 ${ }^{[23]}$ 在分析了 47 个国家本土传播初期的

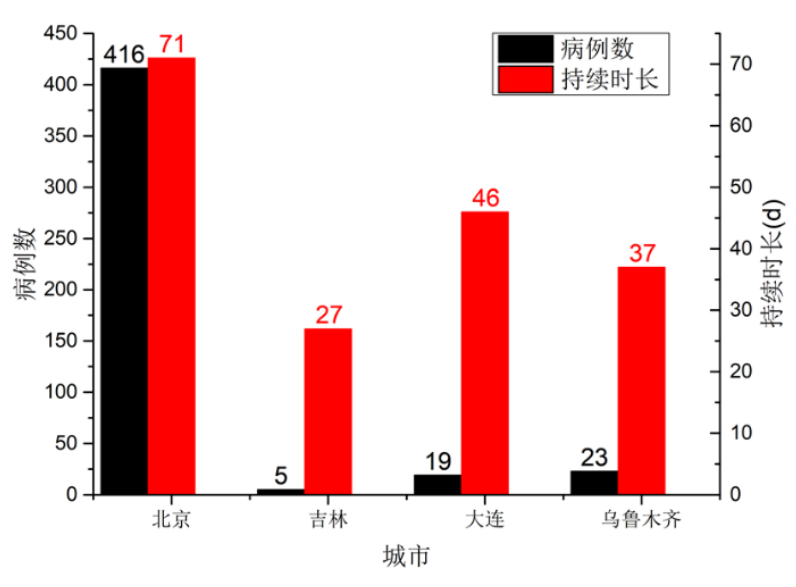

图 2 四个城市第一轮疫情持续时间和累计病例数

数据后, 认为较低的环境温度与新冠疫情在流行早 期加速传播有关。

由此可见, 大部分研究与本研究结论一致, 即 较低的温度有利于 COVID-19 的传播。得到相反结论 的研究或是研究地位于南亚的热带地区, 气候条件 不同; 或包含武汉的数据, 武汉早期由于条件限制, 确诊病例与实际感染病例数有较大出入。

\subsection{2 相对湿度}

对于相对湿度和新冠疫情传播效率之间的关系， 本研究和位于新加坡的研究 ${ }^{[14]}$ 认为两者之间呈正相 关的关系。但除此之外，无论是针对中国多个城市 的研究 ${ }^{[11]}$ 还是位于美国纽约的研究 ${ }^{[17]}$ 均表明, 较低 的相对湿度有利于新冠病毒的传播。这可能是由于, 相对湿度受到气温和气压的影响, 并非一个独立的 变量。并且根据目前的研究, 无法判断温度与相对 湿度对于病毒传播影响的权重; 尤其 Liu 针对中国 多个城市的研究与本研究关于温度的结论是一致的, 而在相对湿度的结论上则相反。我们有理由怀疑, 相对于温度, 湿度可能是影响病毒传播的次要因素。 尚需进一步的研究来得到更强有力的结论。

\subsection{3 风速}

风是传染病传播过程中的一个重要影响因素, 它可以调节各种病原体和媒介的动态 ${ }^{[26]}$ 。目前, 已 有多篇研究涉及到风速对新冠疫情传播的影响 ${ }^{[14 ; 15 ; 17}$ 19; 21], 但其中存在较大的问题: 一是研究区域较少, 多为针对单个城市和中小国家的研究, 其结论不具 有普适性, 还需要针对更多地区的汇总分析研究才 能得到更可靠的结论。 


\section{4. 结论}

本研究对气象因子与北京市两轮疫情的关系提 出了新的见解, 对于加强对新冠肺炎传播现状的认 识, 并制定相关防控措施以防止疫情二次爆发具有 重要意义。本研究的结果可能有助于建立以气象为 基础的新冠肺炎疫情的预测模型, 以帮助政府和医 疗机构及时应对新冠疫情在本地的再次出现。这种 初步评估可以为针对新冠疫情的流行病学的进一步 研究提出假设。

本研究也有一定的局限性。首先, 研究的样本 仅有 4 个国内城市, 还存在病例数和疫情持续时间 较短等问题, 可能会影响统计分析结果。其次, 气 象因素仅仅是影响病毒传播的一个因素, 除此之外, 传染病的传播还受易感人群、卫生基础设施、人们 对社交距离和社交隔离的服从程度等因素影响, 因 此, 开展全面研究需要考虑更多的变量。

\section{致谢}

本论文得到北京市自然科学基金项目（8192026） 支持。

\section{参考文献}

[1]WHO. (2020). Novel Coronavirus(2019-nCoV) Situation Report - 11. Retrieved from https://www.who.int/docs/default-

source/coronaviruse/situation-reports/20200131sitrep-11-ncov.pdf?sfvrsn=de7c0f7_4

[2]WHO. (2020). WHO Director-General's opening remarks at the media briefing on COVID-19 - 11 March 2020. Retrieved from https://www.who.int/dg/speeches/detail/whodirector-general-s-opening-remarks-at-the-mediabriefing-on-covid-19---11-march-2020

[3]WHO. (2020). Novel coronavirus(2019-nCoV) situation reports. Retrieved from https://www.who.int/emergencies/diseases/novelcoronavirus-2019/situation-reports

[4]中华人民共和国国家卫生健康委员会. (2020). 新 型冠状病毒肺炎诊疗方案（试行第八版）. Retrieved

from http://www.nhc.gov.cn/yzygj/s7653p/202008/0a7b df12bd4b46e5bd28ca7f9a7f5e5a.shtml

[5]Tan, J. G., Mu, L. N., Huang, J. X., Yu, S. Z., Chen, B. H., \& Yin, J. (2005). An initial investigation of the association between the SARS outbreak and weather: with the view of the environmental temperature and its variation. Journal of Epidemiology and Community Health, 59(3), 186192. Retrieved from $<$ Go to
ISI>://WOS:000228010200005.

doi:10.1136/jech.2004.020180

[6]Xu, Z., Hu, W., Williams, G., Clements, A. C. A., Kan, H., \& Tong, S. (2013). Air pollution, temperature and pediatric influenza in Brisbane, Australia. Environment International, 59, 384-388. Retrieved from $<$ Go to ISI $>$ ://WOS:000324901000040. doi:10.1016/j.envint.2013.06.022

[7]冯业荣, 朱科伦, 纪忠萍, 杜琳, 王安宇, \& 金顺英. (2005). 广州大气环境因素与 SARS 疫情短期变 化关系的研究. 热带气象学报(02), 191-198.

[8]van Doremalen, N., Bushmaker, T., \& Munster, V. J. (2013). Stability of Middle East respiratory syndrome coronavirus (MERS-CoV) under different environmental conditions. Eurosurveillance, 18(38), 7-10. Retrieved from $<$ Go to $\quad$ ISI $>$ ://WOS:000324726500002. doi:10.2807/1560-7917.Es2013.18.38.20590

[9]Qu, J., \& Wickramasinghe, C. (2017). SARS, MERS and the sunspot cycle. Current Science, 113(8), 1501-1502. Retrieved from $<\mathrm{Go}$ to ISI $>$ ://WOS:000413478800019.

[10]Xie, J., \& Zhu, Y. (2020). Association between ambient temperature and COVID-19 infection in 122 cities from China. Science of the Total Environment, 724. Retrieved from $<\mathrm{Go}$ to ISI>://WOS:000532686000009. doi:10.1016/j.scitotenv.2020.138201

[11]Liu, J., Zhou, J., Yao, J., Zhang, X., Li, L., Xu, X., . . Zhang, K. (2020). Impact of meteorological factors on the COVID-19 transmission: A multi-city study in China. Science of the Total Environment, 726. Retrieved from $<$ Go to ISI>://WOS:000537423900007. doi:10.1016/j.scitotenv.2020.138513

[12]Shi, P., Dong, Y., Yan, H., Zhao, C., Li, X., Liu, W., .. X Xi, S. (2020). Impact of temperature on the dynamics of the COVID-19 outbreak in China. Science of the Total Environment, 728. Retrieved from $<$ Go to ISI $>$ ://WOS:000541009200022. doi:10.1016/j.scitotenv.2020.138890

[13]Yao, Y., Pan, J., Liu, Z., Meng, X., Wang, W., Kan, H., \& Wang, W. (2020). No association of COVID19 transmission with temperature or UV radiation in Chinese cities. European Respiratory Journal, 55(5). Retrieved from $<$ Go to ISI $>$ ://WOS:000531100800019. doi:10.1183/13993003.00517-2020

[14]Pani, S. K., Lin, N.-H., \& RavindraBabu, S. (2020). Association of COVID-19 pandemic with meteorological parameters over Singapore. Science 
of the Total Environment, 740. Retrieved from $<$ Go to ISI $>$ ://WOS:000562379000011. doi:10.1016/j.scitotenv.2020.140112

[15]Ahmadi, M., Sharifi, A., Dorosti, S., Ghoushchi, S. J., \& Ghanbari, N. (2020). Investigation of effective climatology parameters on COVID-19 outbreak in Iran. Science of the Total Environment, 729. Retrieved from $<$ Go to ISI>://WOS:000537441700014. doi:10.1016/j.scitotenv.2020.138705

[16]Tobias, A., \& Molina, T. (2020). Is temperature reducing the transmission of COVID-19? Environmental Research, 186. Retrieved from $<$ Go to ISI $>$ ://WOS:000548186300083. doi:10.1016/j.envres.2020.109553

[17]Bashir, M. F., Ma, B., Bilal, Komal, B., Bashir, M. A., Tan, D., \& Bashir, M. (2020). Correlation between climate indicators and COVID-19 pandemic in New York, USA. Science of the Total Environment, 728. Retrieved from $<$ Go to ISI>://WOS:000541006000007. doi:10.1016/j.scitotenv.2020.138835

[18]Mendez-Arriaga, F. (2020). The temperature and regional climate effects on communitarian COVID19 contagion in Mexico throughout phase 1. Science of the Total Environment, 735. Retrieved from $<$ Go to ISI $>$ ://WOS:000542563500003. doi:10.1016/j.scitotenv.2020.139560

[19]Sahin, M. (2020). Impact of weather on COVID-19 pandemic in Turkey. Science of the Total Environment, 728. Retrieved from $<$ Go to ISI>://WOS:000541006000020. doi:10.1016/j.scitotenv.2020.138810

[20]Tosepu, R., Gunawan, J., Effendy, D. S., Ahmad, L. O. A. I., Lestari, H., Bahar, H., \& Asfian, P. (2020). Correlation between weather and Covid-19 pandemic in Jakarta, Indonesia. Science of the Total Environment, 725. Retrieved from $<$ Go to ISI>://WOS:000537428300014. doi:10.1016/j.scitotenv.2020.138436

[21]Menebo, M. M. (2020). Temperature and precipitation associate with Covid-19 new daily cases: A correlation study between weather and Covid-19 pandemic in Oslo, Norway. Science of the Total Environment, 737. Retrieved from $<$ Go to ISI>://WOS:000553723000003. doi:10.1016/j.scitotenv.2020.139659

[22]Wu, F., Zhao, S., Yu, B., Chen, Y.-M., Wang, W., Song, Z.-G., . . . Zhang, Y.-Z. (2020). A new coronavirus associated with human respiratory disease in China (vol 579, pg 265, 2020). Nature, 580(7803), E7-E7. Retrieved from $<$ Go to
ISI $>$ ://WOS:000522805300001. doi:10.1038/s41586-020-2202-3

[23]Holtmann, M., Jones, M., Shah, A., \& Holtmann, G. (2020). Low ambient temperatures are associated with more rapid spread of COVID-19 in the early phase of the endemic. Environmental Research, 186. Retrieved from $<$ Go to ISI>://WOS:000548186300125. doi:10.1016/j.envres.2020.109625

[24] 北京市人民政府．(2019). 北京市气候简介. Retrieved from http://www.beijing.gov.cn/renwen/bjgk/bjgk/qh/20 1911/t20191106_1872564.html

[25]McMichael, A. J., Wilkinson, P., Kovats, R. S., Pattenden, S., Hajat, S., Armstrong, B., . . . Nikiforov, B. (2008). International study of temperature, heat and urban mortality: the 'ISOTHURM' project. International Journal of Epidemiology, 37(5), 1121-1131. Retrieved from $<$ Go to ISI $>$ ://WOS:000259771500028. doi:10.1093/ije/dyn086

[26]Ellwanger, J. H., \& Chies, J. A. B. (2018). Wind: a neglected factor in the spread of infectious diseases. Lancet Planetary Health, 2(11), E475-E475. Retrieved from $<$ Go to ISI>://WOS:000525878300008. doi:10.1016/s2542-5196(18)30238-9 